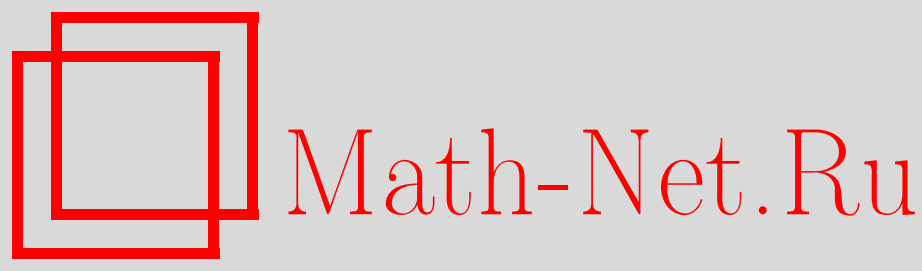

Л. М. Сладь, К вопросу об электромагнитных свойствах недираковских частиц со спином покоя $1 / 2, T M \Phi, 2010$, том 165, номер 1, 48-69

DOI: https://doi.org/10.4213/tmf6563

Использование Общероссийского математического портала Math-Net.Ru подразумевает, что вы прочитали и согласны с пользовательским соглашением http: //www . mathnet.ru/rus/agreement

Параметры загрузки:

IP : 35.174 .16 .151

26 апреля 2023 г., 17:19:41

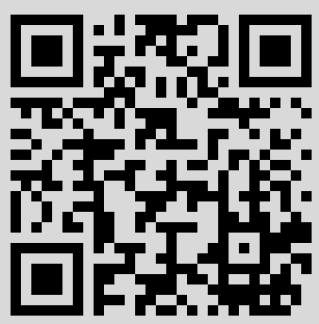




\section{К ВОПРОСУ ОБ ЭЛЕКТРОМАГНИТНЫХ СВОЙСТВАХ НЕДИРАКОВСКИХ ЧАСТИЦ СО СПИНОМ ПОКОЯ $1 / 2$}

Решен ряд вопросов, связанных с аналитическим описанием электромагнитных формфакторов недираковских частиц со спином покоя $1 / 2$. Найдена общая структура матричного антисимметричного тензорного оператора. Получены два рекуррентных соотношения для матричных элементов конечных преобразований собственной группы Лоренца и явные формулы для некоторого множества таких элементов. В рамках теории полей с двойной симметрией обсуждена запись компонент волновых векторов частиц в виде бесконечных непрерывных дробей. Показано, что при $Q^{2} \leqslant 0.5(\Gamma \ni \mathrm{B} / \mathrm{c})^{2}$, где $Q^{2}-$ квадрат переданного импульса, можно, не прибегая к явному введению внутренней структуры частицы, получить падающие с ростом $Q^{2}$ электромагнитные формакторы, близкие к тем, которые экспериментально наблюдаются у протона.

Ключевые слова: электромагнитные формфакторы, тензорные операторы, конечные лоренцевы преобразования, бесконечные непрерывные дроби.

\section{1. ВВЕДЕНИЕ}

Различие между протоном и электроном, проявляющееся в их электромагнитном взаимодействии, получило свое первое свидетельство в результате измерения магнитного момента протона, проведенного в 1933 г. [1], а окончательно утвердилось после опытов МакАллистера-Хофштадтера по упругому рассеянию электронов на протонах [2]. Суть этого различия можно свести к неприемлемости для протона одной или нескольких характеристик электрона из числа трех следующих. Во-первых, электрону сопоставляется дираковское представление собственной группы Лоренца $L_{+}^{\uparrow}$. Во-вторых, вплоть до экспериментально достижимых размеров порядка $10^{-16}$ см электрон проявляет себя как точечная частица, что соответствует локальности лагранжиана его взаимодействия с фотоном, при которой поля всех частиц берутся в одной и той же точке. В-третьих, лагранжиан электромагнитного взаимодействия электрона является минимальным: он, выражаясь через вектор-потенциал электромагнитного поля $A^{\mu}$, не содержит производных от него.

${ }^{*}$ Научно-исследовательский институт ядерной физики им. Д. В. Скобельцына, Московский государственный университет им. М. В. Ломоносова, Москва, Россия.

E-mail: slad@theory.sinp.msu.ru 
Сложившаяся в дальнейшем теоретическая концепция протона и нейтрона апеллирует к их неминимальному электромагнитному взаимодействию и к сложной внутренней структуре, но практически не подвергает сомнению предположение о том, что нуклон является дираковской частицей. Это находит свое отражение в общем виде нуклонного электромагнитного тока в импульсном пространстве [3]:

$$
j^{\mu}\left(p, p_{0}\right)=i e \bar{u}(p)\left[\gamma^{\mu} F_{1}\left(Q^{2}\right)+\frac{i \kappa}{2 M} \sigma^{\mu \nu} q_{\nu} F_{2}\left(Q^{2}\right)\right] u\left(p_{0}\right),
$$

где $\bar{u}$ и $u$ - дираковские спиноры, $\kappa$ - аномальный магнитный момент, $M$ - масса нуклона, $q=p-p_{0}, Q^{2}=-q^{2}$. Наряду с формфакторами Дирака $F_{1}$ и Паули $F_{2}$ из формулы (1) вводят формфакторы Сакса $G_{\mathrm{E}}$ и $G_{\mathrm{M}}$, которые задаются соотношениями [4]

$$
G_{\mathrm{E}}=F_{1}-\kappa \tau F_{2}, \quad G_{\mathrm{M}}=F_{1}+\kappa F_{2},
$$

где $\tau=Q^{2} / 4 M^{2}$, и которые, как считают, описывают распределения электрического заряда и магнетизма нуклона. Использование величин $G_{\mathrm{E}}$ и $G_{\mathrm{M}}$ приводит формулу Розенблюта [5], описывающую упругое рассеяние неполяризованных электронов на неполяризованных нуклонах в лабораторной системе отсчета, к наиболее простому виду

$$
\frac{d \sigma}{d \Omega}=\frac{\alpha^{2} E^{\prime} \cos ^{2}(\theta / 2)}{4 E^{3} \sin ^{4}(\theta / 2)}\left[\frac{G_{\mathrm{E}}^{2}+\tau G_{\mathrm{M}}^{2}}{1+\tau}+2 \tau G_{\mathrm{M}}^{2} \operatorname{tg}^{2} \frac{\theta}{2}\right],
$$

где $E$ и $E^{\prime}$ - энергия электрона в начальном и конечном состояниях соответственно, $\theta$ - угол рассеяния электрона. Обстоятельный обзор результатов экспериментальных измерений электромагнитных формфакторов протона и нейтрона и их разнообразной теоретической интерпретации можно найти в статье [6].

Отказ от описания протона дираковским спинором влечет за собой многочисленные неопределенности. Один тип неопределенностей обусловлен тем, что частице, обладающей в системе покоя спином $1 / 2$, можно поставить в соответствие бесконечное количество неприводимых представлений собственной группы Лоренца $L_{+}^{\uparrow}$ вида ${ }^{1)}\left(-1 / 2, l_{1}\right)$ и $\left(1 / 2, l_{1}\right)$, где $l_{1}$ - произвольное комплексное число, и бесконечное количество построенных из них приводимых представлений. Другой тип неопределенностей порождается произволом в константах полевой теории, допускаемым ее релятивистской инвариантностью [7], [8], причем этот произвол может быть бесконечным, если рассматриваются, например, поля класса ISFIR, которые преобразуются по представлениям, разложимым в бесконечную прямую сумму конечномерных неприводимых представлений группы $L_{+}^{\uparrow}$.

Аргументы в пользу возможности описания адронов полями класса ISFIR приведены в работах [9], [10]. Отметим, что исследования теории таких полей не проводились до тех пор, пока не появился симметрийный подход к устранению бесконечного

\footnotetext{
1) Неприводимые представления группы $L_{+}^{\uparrow}$ обозначают [7], [8] двумя индексами $\left(l_{0}, l_{1}\right)$, где $2 l_{0}$ - целое число, а $l_{1}$ - произвольное комплексное число. Канонический базис пространства представления $\left(l_{0}, l_{1}\right)$ связывают с подгруппой $S O(3)$ и обозначают через $\xi_{\left(l_{0}, l_{1}\right) l m}$, где $l-$ спин, a $m$ - его проекция на третью ось, причем $m=-l,-l+1, \ldots, l$ и $l=\left|l_{0}\right|,\left|l_{0}\right|+1, \ldots$. В общем случае последовательность значений $l$ бесконечна. Представление $\left(l_{0}, l_{1}\right)$ конечномерно, а выписанная последовательность спинов обрывается на числе $\left|l_{1}\right|-1$, если $2 l_{0}$ и $2 l_{1}$ являются целыми числами одинаковой четности, причем $\left|l_{1}\right|>\left|l_{0}\right|$. Дираковское представление - это $(-1 / 2,3 / 2) \oplus(1 / 2,3 / 2)$.
} 
произвола в соответствующих лагранжианах. Общее определение понятия двойной симметрии [11], включающее в себя как частные случаи симметрию $\sigma$-модели Гелл-Манна-Леви [12] и суперсимметрию, позволило эффективно дополнить [9] релятивистскую инвариантность (первичную симметрию) теории полей класса ISFIR требованием ее дополнительной (вторичной) симметрии, порождаемой преобразованиями вида

$$
\psi(x) \rightarrow \psi^{\prime}(x)=\exp \left[-i D^{\mu} \theta_{\mu}\right] \psi(x)
$$

где параметры $\theta_{\mu}$ являются компонентами полярного или аксиального 4-вектора ортохронной группы Лоренца $L^{\uparrow}$, а величины $D^{\mu}$ - матричными операторами. Чтобы избежать бесконечного вырождения по спину в спектре масс получаемой теории, обусловленного расширением группы Лоренца, вводится [13] спонтанное нарушение вторичной симметрии. В итоге, как показано в работе [14], построенные таким образом свободные релятивистски-инвариантные уравнения фермионных полей класса ISFIR дают спектры масс, которые находятся в согласии с экспериментальной картиной барионных резонансов и с партонной моделью адронов, дополняемой гипотезой о конфайнменте.

Стоит подчеркнуть, что все рассматривавшиеся прежде релятивистски-инвариантные теории свободных полей с бесконечным числом степеней свободы - билокальные уравнения (см. обзорную статью [15]) и уравнения типа Гельфанда-Яглома [7], [8] (с уточнениями следствий в работе [16]) для полей класса FSIIR, которые преобразуются по представлениям, разложимым в конечную прямую сумму бесконечномерных неприводимых представлений группы $L_{+}^{\uparrow}$, - обладали непригодными для физики частиц спектрами масс с точкой сгущения в нуле. Бесконечное количество состояний в теории полей класса ISFIR с двойной симметрией, по-видимому, отражает некоторую внутреннюю структуру соответствующих частиц, а физически вполне удовлетворительные спектры масс теории свидетельствуют о том, что предлагаемый нами подход к описанию какого-либо адрона как целого бесконечнокомпонентным монолокальным полем в основных чертах правилен. Теперь хотелось бы выяснить, как влияет на физическое содержание существующих структурных характеристик частиц со спином покоя $1 / 2$ переход от дираковского представления группы $L_{+}^{\uparrow}$ к недираковскому, сопоставимому частице как целому, и можно ли получить удовлетворительное описание экспериментальных процессов с участием составных частиц на основании рассмотрения локального взаимодействия монолокальных полей, не прибегая к явному введению структурных величин.

\section{2. ОБЩЕЕ ОПИСАНИЕ ЭЛЕКТРОМАГНИТНОГО ТОКА И ФОРМФАКТОРОВ НЕДИРАКОВСКИХ ЧАСТИЦ СО СПИНОМ ПОКОЯ $1 / 2$}

Первые общие результаты отказа от описания нуклона дираковским спинором, касающиеся его электромагнитных свойств, получены в недавней работе [10]. Для широкого класса представлений собственной группы Лоренца, сопоставляемых полю $\psi$ частицы со спином покоя $1 / 2$ и массой $M$, полярный 4-вектор электромагнитного тока, не содержащий структурных функций от квадрата переданного импульса, 
можно записать в следующем виде:

$$
\mathcal{J}^{\mu}\left(p, p_{0}\right)=i e\left(\psi(p),\left[Q_{0} \Gamma^{\mu}+M^{-1} \Lambda^{\mu \nu}\left(p, p_{0}\right) q_{\nu}\right] \psi\left(p_{0}\right)\right)
$$

где

$\Lambda^{\mu \nu}\left(p, p_{0}\right) \equiv \Gamma^{\mu \nu}+M^{-1} a_{i_{1}} \Gamma^{\mu \nu \nu_{1}}\left(p_{i_{1}}\right)_{\nu_{1}}+\cdots+M^{-j} a_{i_{1} \ldots i_{j}} \Gamma^{\mu \nu \nu_{1} \ldots \nu_{j}}\left(p_{i_{1}}\right)_{\nu_{1}} \ldots\left(p_{i_{j}}\right)_{\nu_{j}}+\cdots$,

$\left(\psi_{1}, \psi_{2}\right)$ - релятивистски-инвариантная билинейная форма, которую мы считаем невырожденной, $Q_{0}$ - заряд частицы в единицах заряда позитрона $e, \Gamma^{\mu}-4$-векторный матричный оператор, $\Gamma^{\mu \nu}$ и $\Gamma^{\mu \nu \nu_{1} \ldots \nu_{j}}-$ антисимметричные по индексам $\mu$ и $\nu$ бесследные матричные тензорные операторы группы Лоренца, $a_{i_{1} \ldots i_{j}}$ - числовые коэффициенты, а $p_{k} \in\left\{p_{0}, p\right\}$ для любого индекса $k$. При этом предполагается, что полевой вектор $\psi(p)$ подчиняется некоторому релятивистски-инвариантному уравнению

$$
\left(\Gamma^{\mu} p_{\mu}-R\right) \psi(p)=0,
$$

где $R$ - скалярный матричный оператор.

Членам в соотношениях (5) и (6), содержащим 4-импульс $p_{k}$, в координатном представлении лагранжиана электромагнитного взаимодействия отвечают члены с производными от поля $\psi(x)$, которые можно трактовать как высшие электрические мультиполи составной частицы. Вместо множества 4-импульсов $\left\{p_{0}, p\right\}$ в (5) и (6) удобнее рассматривать множество $\left\{p_{0}, q\right\}$.

В работе [10] показано, что угловое распределение конечных частиц в процессе упругого рассеяния неполяризованных электронов на неполяризованных частицах со спином покоя $1 / 2$ независимо от приписываемого им представления $S_{0}$ группы $L_{+}^{\uparrow}$ дается формулой Розенблюта $(3)$, в которой роль электромагнитных факторов играют следующие величины:

$$
\begin{aligned}
G_{\mathrm{E}} & =\frac{C}{\sqrt{\tau+1}}\left(\psi_{\frac{1}{2}}(p),\left[Q_{0} R-q^{3} \Lambda^{03}\right] \psi_{\frac{1}{2}}\left(p_{0}\right)\right), \\
G_{\mathrm{M}} & =\frac{C}{\sqrt{\tau}}\left(\psi_{\frac{1}{2}}(p),\left[Q_{0} M \Gamma^{1}+q^{0} \Lambda^{10}-q^{3} \Lambda^{13}\right] \psi_{-\frac{1}{2}}\left(p_{0}\right)\right),
\end{aligned}
$$

где

$$
C=\left(\psi_{\frac{1}{2}}\left(p_{0}\right), R \psi_{\frac{1}{2}}\left(p_{0}\right)\right)^{-1}
$$

Величины $G_{\mathrm{E}}$ и $G_{\mathrm{M}}$, задаваемые этими формулами, являются константами для дираковских частиц и нетривиальными функциями от квадрата переданного импульса $Q^{2}$ для недираковских частиц со спином покоя $1 / 2$. Конкретный вид функций $G_{\mathrm{E}}\left(Q^{2}\right)$ и $G_{\mathrm{M}}\left(Q^{2}\right)$ определяется целым рядом обстоятельств: во-первых, выбором представления $S_{0}$; во-вторых, разложением волнового вектора $\psi\left(p_{0}\right)$ частицы в ее системе покоя по векторам канонического базиса пространства представления $S_{0}$, зависящим от структуры операторов уравнения (7); в-третьих, константами операторов $\Gamma^{\mu \nu}$ и $\Gamma^{\mu \nu \nu_{1} \ldots \nu_{j}}, j=1,2, \ldots$. Ни одно из этих обстоятельств в явном виде не зависит от внутренней структуры той или иной недираковской частицы со спином покоя $1 / 2$, и потому можно сказать, что соответствующие формфакторы $G_{\mathrm{E}}$ 
и $G_{\mathrm{M}}$, входящие в формулу Розенблюта $(3)$, задаются внешними характеристиками частицы как целого.

Далее мы приводим отсутствующее в литературе описание ряда величин общей релятивистской теории поля, необходимых для вычислений формфакторов (8), (9) недираковских частиц со спином покоя $1 / 2$. Мы находим общую структуру матричных антисимметричных тензорных операторов второго ранга и приводим явный вид некоторых матричных элементов конечных преобразований собственной группы Лоренца для конечномерных неприводимых представлений $\left( \pm 1 / 2, l_{1}\right)$ (ранее подобные элементы были найдены только для унитарных (бесконечномерных) неприводимых представлений [17]). Наряду с этим мы рассматриваем относящиеся к току (5), (6) задачи, для которых решения даются в рамках теории бесконечнокомпонентных полей класса ISFIR с двойной симметрией. Именно, мы приводим результаты устранения бесконечного произвола в константах оператора $\Gamma^{\mu \nu}$, обсуждаем структуру тензорных операторов третьего и более высокого рангов $\Gamma^{\mu \nu \nu_{1} \ldots \nu_{j}}$, $j=1,2, \ldots$, и предлагаем запись компонент волновых векторов, удовлетворяющих уравнению (7), в виде бесконечных непрерывных дробей.

В теории полей класса ISFIR с двойной симметрией электромагнитный ток (5), (6) в общем случае содержит бесконечное число матричных операторов и тем самым бесконечное число произвольных (нормировочных) констант. Это лишает такое описание электромагнитного взаимодействия недираковских частиц простоты и изящества. Мы пока не видим никакого симметрийного способа устранить бесконечный произвол в константах рассматриваемого тока. Тем не менее, ограничиваясь в токе (5), (6) тензорными операторами четвертого ранга и значениями квадрата переданного импульса, не превышающими $0.5(\Gamma \ni \mathrm{B} / \mathrm{c})^{2}$, мы приходим на основании численных расчетов к принципиальному заключению о возможности получения электромагнитных формфакторов, убывающих с ростом $Q^{2}$, без явного привлечения внутренней структуры частиц со спином покоя $1 / 2$.

\section{3. ОБЩАЯ СТРУКТУРА МАТРИЧНЫХ АНТИСИММЕТРИЧНЫХ ТЕНЗОРНЫХ ОПЕРАТОРОВ ВТОРОГО РАНГА}

Пусть элементу $g$ собственной группы Лоренца соответствует следующее преобразование ковариантных компонент 4-вектора:

$$
x_{\mu}^{\prime}=[l(g)]_{\mu}{ }^{\nu} x_{\nu}
$$

Тогда соответствующее преобразование ковариантных компонент антисимметричного тензора второго ранга можно записать в виде

$$
y_{\mu \nu}^{\prime}=[T(g)]_{\mu \nu}^{\alpha \beta} y_{\alpha \beta},
$$

где

$$
[T(g)]_{\mu \nu}{ }^{\alpha \beta}=\frac{[l(g)]_{\mu}{ }^{\alpha}[l(g)]_{\nu}{ }^{\beta}-[l(g)]_{\nu}{ }^{\alpha}[l(g)]_{\mu}{ }^{\beta}}{2} .
$$

Матричные операторы $K^{\mu \nu}$, действующие на поля $\psi$ как на векторы пространства представления $S(g)$ собственной группы Лоренца, образуют антисимметричный оператор, если величина $\left(\psi_{1}, K^{\mu \nu} \psi_{2}\right)$ преобразуется относительно группы $L_{+}^{\uparrow}$ 
как антисимметричный тензор, т. е. свертка $\left(\psi_{1}, K^{\mu \nu} y_{\mu \nu} \psi_{2}\right)$ представляет собой инвариантную невырожденную билинейную форму. Отсюда следует, что операторы $K^{\mu \nu}$ должны удовлетворять такому условию:

$$
K^{\mu \nu}=S^{-1}(g) K^{\alpha \beta} S(g)[T(g)]_{\alpha \beta}^{\mu \nu} .
$$

Бесконечно малые собственные преобразования Лоренца 4-вектора и вектора в пространстве представления $S(g)$ вводят [7], соответственно, в виде

$$
x_{\mu}^{\prime}=x_{\mu}+\epsilon_{\mu}{ }^{\nu} x_{\nu}, \quad \psi^{\prime}=\psi+\frac{1}{2} \epsilon_{\mu \nu} I^{\mu \nu} \psi
$$

где параметры преобразований $\epsilon_{\mu \nu}$ и инфинитезимальные операторы $I^{\mu \nu}$ антисимметричны: $\epsilon_{\mu \nu}=-\epsilon_{\nu \mu}, I^{\mu \nu}=-I^{\nu \mu}$.

Из формул (11), (13)-(15) следует, что должны выполняться следующие коммутационные соотношения:

$$
\left[I^{\mu \nu}, K^{\alpha \beta}\right]=-g^{\mu \alpha} K^{\nu \beta}+g^{\mu \beta} K^{\nu \alpha}+g^{\nu \alpha} K^{\mu \beta}-g^{\nu \beta} K^{\mu \alpha}
$$

где $g^{00}=-g^{11}=-g^{22}=-g^{33}=1, g^{\gamma \delta}=0$ при $\gamma \neq \delta$.

Отметим, что коммутационные соотношения для инфинитезимальных операторов получаются из (16) заменой $K^{\gamma \delta} \rightarrow I^{\gamma \delta}$. Матричная реализация операторов $I^{\mu \nu}$ в пространстве любого неприводимого представления группы $L_{+}^{\uparrow}$ известна (см. paботы [7], [8] или статью [9]).

Среди 36 условий (16) имеется только 8 независимых. Их можно выбрать так:

$$
\left[I^{03}, K^{03}\right]=0, \quad\left[I^{12}, K^{03}\right]=0, \quad\left[I^{01}, K^{31}\right]=K^{03}
$$

И

$$
\begin{gathered}
{\left[I^{01}, K^{03}\right]=K^{31}, \quad\left[I^{02}, K^{03}\right]=-K^{23}, \quad\left[I^{31}, K^{03}\right]=K^{01}} \\
{\left[I^{23}, K^{03}\right]=-K^{02}, \quad\left[I^{23}, K^{31}\right]=K^{12}}
\end{gathered}
$$

Остальные 28 условий (16) являются следствием равенств (17), (18) и коммутационных соотношений для операторов $I^{\mu \nu}$. Подставив в (17) оператор $K^{31}$, выраженный через $K^{03}$ с помощью первого из равенств (18), мы получаем три независимых уравнения, которым должен подчиняться оператор $K^{03}$. Найдя $K^{03}$ и используя формулы (18), мы получаем все компоненты антисимметричного оператора $K^{\mu \nu}$.

Решить систему уравнений (17) относительно неизвестного оператора $K^{03}$ означает указать результат действия последнего на векторы канонического базиса $\xi_{\left(l_{0}, l_{1}\right) l m}$ любого неприводимого представления $\left(l_{0}, l_{1}\right)$ собственной группы Лоренца. Принимая во внимание известное действие операторов $I^{12}, I^{03}$ и $I^{01}$ на базисные векторы 
$\xi_{\left(l_{0}, l_{1}\right) l m}$, находим

$$
\begin{aligned}
& K^{03} \xi_{\left(l_{0}, l_{1}\right) l m}=f\left(l_{0}-1, l_{1}-1 ; l_{0}, l_{1}\right)\left[a(l, m) \sqrt{\left(l+l_{0}-1\right)\left(l+l_{0}\right)\left(l+l_{1}-1\right)\left(l+l_{1}\right)} \times\right. \\
& \times \xi_{\left(l_{0}-1, l_{1}-1\right)(l-1) m}-b(l, m) \sqrt{\left(l-l_{0}+1\right)\left(l+l_{0}\right)\left(l-l_{1}+1\right)\left(l+l_{1}\right)} \xi_{\left(l_{0}-1, l_{1}-1\right) l m}- \\
& \left.-a(l+1, m) \sqrt{\left(l-l_{0}+1\right)\left(l-l_{0}+2\right)\left(l-l_{1}+1\right)\left(l-l_{1}+2\right)} \xi_{\left(l_{0}-1, l_{1}-1\right)(l+1) m}\right]+ \\
& +f\left(l_{0}-1, l_{1}+1 ; l_{0}, l_{1}\right)\left[a(l, m) \sqrt{\left(l+l_{0}-1\right)\left(l+l_{0}\right)\left(l-l_{1}-1\right)\left(l-l_{1}\right)} \xi_{\left(l_{0}-1, l_{1}+1\right)(l-1) m}+\right. \\
& +b(l, m) \sqrt{\left(l-l_{0}+1\right)\left(l+l_{0}\right)\left(l-l_{1}\right)\left(l+l_{1}+1\right)} \xi_{\left(l_{0}-1, l_{1}+1\right) l m}- \\
& \left.-a(l+1, m) \sqrt{\left(l-l_{0}+1\right)\left(l-l_{0}+2\right)\left(l+l_{1}+1\right)\left(l+l_{1}+2\right)} \xi_{\left(l_{0}-1, l_{1}+1\right)(l+1) m}\right]+ \\
& +f\left(l_{0}+1, l_{1}-1 ; l_{0}, l_{1}\right)\left[a(l, m) \sqrt{\left(l-l_{0}-1\right)\left(l-l_{0}\right)\left(l+l_{1}-1\right)\left(l+l_{1}\right)} \xi_{\left(l_{0}+1, l_{1}-1\right)(l-1) m}+\right. \\
& +b(l, m) \sqrt{\left(l-l_{0}\right)\left(l+l_{0}+1\right)\left(l-l_{1}+1\right)\left(l+l_{1}\right)} \xi_{\left(l_{0}+1, l_{1}-1\right) l m}- \\
& \left.-a(l+1, m) \sqrt{\left(l+l_{0}+1\right)\left(l+l_{0}+2\right)\left(l-l_{1}+1\right)\left(l-l_{1}+2\right)} \xi_{\left(l_{0}+1, l_{1}-1\right)(l+1) m}\right]+ \\
& +f\left(l_{0}+1, l_{1}+1 ; l_{0}, l_{1}\right)\left[a(l, m) \sqrt{\left(l-l_{0}-1\right)\left(l-l_{0}\right)\left(l-l_{1}-1\right)\left(l-l_{1}\right)} \xi_{\left(l_{0}+1, l_{1}+1\right)(l-1) m}-\right. \\
& -b(l, m) \sqrt{\left(l-l_{0}\right)\left(l+l_{0}+1\right)\left(l-l_{1}\right)\left(l+l_{1}+1\right)} \xi_{\left(l_{0}+1, l_{1}+1\right) l m}- \\
& \left.-a(l+1, m) \sqrt{\left(l+l_{0}+1\right)\left(l+l_{0}+2\right)\left(l+l_{1}+1\right)\left(l+l_{1}+2\right)} \xi_{\left(l_{0}+1, l_{1}+1\right)(l+1) m}\right]+ \\
& +f_{1}\left(l_{0}, l_{1} ; l_{0}, l_{1}\right)\left[a(l, m) \sqrt{\left(l-l_{0}\right)\left(l+l_{0}\right)\left(l-l_{1}\right)\left(l+l_{1}\right)} \xi_{\left(l_{0}, l_{1}\right)(l-1) m}-b(l, m) l_{0} l_{1} \xi_{\left(l_{0}, l_{1}\right) l m}-\right. \\
& \left.-a(l+1, m) \sqrt{\left(l-l_{0}+1\right)\left(l+l_{0}+1\right)\left(l-l_{1}+1\right)\left(l+l_{1}+1\right)} \xi_{\left(l_{0}, l_{1}\right)(l+1) m}\right]+ \\
& +f_{2}\left(l_{0}, l_{1} ; l_{0}, l_{1}\right) m \xi_{\left(l_{0}, l_{1}\right) l m},
\end{aligned}
$$

где

$$
a(l, m)=\frac{1}{l} \sqrt{\frac{(l-m)(l+m)}{4 l^{2}-1}}, \quad b(l, m)=\frac{m}{l(l+1)},
$$

а величины $f\left(l_{0} \pm 1, l_{1}-1 ; l_{0}, l_{1}\right), f\left(l_{0} \pm 1, l_{1}+1 ; l_{0}, l_{1}\right)$ и $f_{j}\left(l_{0}, l_{1} ; l_{0}, l_{1}\right), j=1,2$, являются произвольными константами.

В семействе антисимметричных тензорных операторов $K^{\mu \nu}$ можно выделить два типа операторов, преобразующихся по неэквивалентным неприводимым представлениям ортохронной группы Лоренца. Мы будем обозначать их через $\Gamma^{\mu \nu}$ и $L^{\mu \nu}$, полагая, что свертки этих операторов с двумя произвольными полярными 4-векторами дают соответственно скалярный и псевдоскалярный операторы группы $L^{\uparrow}$. Это означает, что трансформационные свойства антисимметричных операторов этих двух типов относительно пространственного отражения $P$ описываются соотношениями

$$
P K^{0 i}+\eta K^{0 i} P=0, \quad P K^{j k}-\eta K^{j k} P=0, \quad i, j, k=1,2,3,
$$

где $\eta=1$, если $K^{\mu \nu}=\Gamma^{\mu \nu}$, и $\eta=-1$, если $K^{\mu \nu}=L^{\mu \nu}$.

Поскольку (см. работы [7], [8])

$$
P \xi_{\left(l_{0}, l_{1}\right) l m}= \pm(-1)^{[l]} \xi_{\left(-l_{0}, l_{1}\right) l m}
$$

где знак плюс или минус берется одним и тем же для всех неприводимых представлений группы $L_{+}^{\uparrow}$, принадлежащих данному представлению $S_{0}$, то из соотношений 
(19)-(21) получаем следующие связи между константами операторов $\Gamma^{\mu \nu}$ и $L^{\mu \nu}$ :

$$
\begin{aligned}
f\left(l_{0} \pm 1, l_{1}-1 ; l_{0}, l_{1}\right) & =\eta f\left(-l_{0} \mp 1, l_{1}-1 ;-l_{0}, l_{1}\right), \\
f\left(l_{0} \pm 1, l_{1}+1 ; l_{0}, l_{1}\right) & =\eta f\left(-l_{0} \mp 1, l_{1}+1 ;-l_{0}, l_{1}\right), \\
f_{1}\left(l_{0}, l_{1} ; l_{0}, l_{1}\right) & =\eta f_{1}\left(-l_{0}, l_{1} ;-l_{0}, l_{1}\right) \\
f_{2}\left(l_{0}, l_{1} ; l_{0}, l_{1}\right) & =-\eta f_{2}\left(-l_{0}, l_{1} ;-l_{0}, l_{1}\right) .
\end{aligned}
$$

В электромагнитный ток (5), (6) входит антисимметричный тензорный оператор второго ранга типа $\Gamma^{\mu \nu}$.

\section{4. АНТИСИММЕТРИЧНЫЕ ТЕНЗОРНЫЕ ОПЕРАТОРЫ В ТЕОРИИ ПОЛЕЙ КЛАССА ISFIR С ДВОЙНОЙ СИММЕТРИЕЙ}

Чтобы при рассмотрении электромагнитного тока (5), (6) в рамках теории полей класса ISFIR избавиться от бесконечного произвола в константах оператора $\Gamma^{\mu \nu}$, будем полагать, что этот ток инвариантен относительно преобразований вторичной симметрии (4), вследствие чего должны выполняться условия

$$
\left[D^{\alpha}, \Gamma^{\mu \nu}\right]=0
$$

Среди 24 соотношений (24) имеются только два независимых

$$
\left[D^{0}, \Gamma^{03}\right]=0, \quad\left[D^{0}, \Gamma^{12}\right]=0,
$$

а остальные 22 условия (24) являются следствием выписанных двух, а также соотношений (16) и коммутационных соотношений 4-векторного оператора с инфинитезимальными операторами группы $L_{+}^{\uparrow}$ (см. работы [7]-[9]).

Из счетного множества вариантов дважды симметричной теории полей класса ISFIR со спонтанно нарушенной вторичной симметрией [9], [13] мы выбираем для дальнейшего изучения один, который является наиболее простым и характеризуется физически приемлемым спектром масс [14]. В выбираемом варианте теории, во-первых, поле преобразуется по представлению собственной группы Лоренца, состоящему из всех тех и только тех неприводимых конечномерных представлений, которые содержат спин $1 / 2$ :

$$
S^{3 / 2}=\bigoplus_{N=1}^{+\infty}\left[\left(-\frac{1}{2}, \frac{1}{2}+N\right) \oplus\left(\frac{1}{2}, \frac{1}{2}+N\right)\right] .
$$

Во-вторых, преобразования вторичной симметрии (4) образуют четырехпараметрическую абелеву группу: $\left[D^{\mu}, D^{\nu}\right]=0$. В-третьих, полярные 4-векторные операторы $D^{\mu}$ из преобразований (4) и $\Gamma^{\mu}$ из уравнения (7) с точностью до постоянного числового множителя совпадают друг с другом, причем

$$
\begin{aligned}
& d_{0}^{-1} D^{0} \xi_{\left( \pm \frac{1}{2}, l_{1}\right) l m}=c_{0}^{-1} \Gamma^{0} \xi_{\left( \pm \frac{1}{2}, l_{1}\right) l m}=\frac{l+1 / 2}{l_{1}^{2}-1 / 4} \xi_{\left(\mp \frac{1}{2}, l_{1}\right) l m}- \\
& -\frac{\sqrt{\left(l_{1}-l-1\right)\left(l_{1}+l\right)}}{l_{1}-1 / 2} \xi_{\left( \pm \frac{1}{2}, l_{1}-1\right) l m}-\frac{\sqrt{\left(l_{1}-l\right)\left(l_{1}+l+1\right)}}{l_{1}+1 / 2} \xi_{\left( \pm \frac{1}{2}, l_{1}+1\right) l m},
\end{aligned}
$$


где $d_{0}$ и $c_{0}$ - произвольные действительные константы. В-четвертых, существуют только два (с точностью до числового множителя) 4-векторных оператора, компоненты которых коммутируют с компонентами оператора $D^{\mu}$ : сам оператор $D^{\mu}$ и аксиальный 4-векторный оператор $L^{\mu}$, временная компонента которого действует на векторы канонического базиса следующим образом:

$$
\begin{aligned}
& b_{0}^{-1} L^{0} \xi_{\left( \pm \frac{1}{2}, l_{1}\right) l m}=\mp \frac{l_{1}(l+1 / 2)}{l_{1}^{2}-1 / 4} \xi_{\left(\mp \frac{1}{2}, l_{1}\right) l m} \pm \\
& \quad \pm \frac{\sqrt{\left(l_{1}-l-1\right)\left(l_{1}+l\right)}}{2 l_{1}-1} \xi_{\left( \pm \frac{1}{2}, l_{1}-1\right) l m} \mp \frac{\sqrt{\left(l_{1}-l\right)\left(l_{1}+l+1\right)}}{2 l_{1}+1} \xi_{\left( \pm \frac{1}{2}, l_{1}+1\right) l m},
\end{aligned}
$$

где $b_{0}-$ произвольная константа.

Как показывают прямые вычисления, существует только один (с точностью до числового множителя) антисимметричный тензорный оператор $\Gamma^{\mu \nu}$, удовлетворяющий условиям (25) с учетом соотношений $(19),(20),(23)$ (при $\eta=1$ ) и (27). Значения его констант таковы:

$$
\begin{aligned}
f\left( \pm \frac{1}{2}, l_{1}+1 ; \mp \frac{1}{2}, l_{1}\right) & =f\left( \pm \frac{1}{2}, l_{1} ; \mp \frac{1}{2}, l_{1}+1\right)=\frac{i h_{0}}{l_{1}+1 / 2} \\
f_{1}\left( \pm \frac{1}{2}, l_{1} ; \pm \frac{1}{2}, l_{1}\right) & =\frac{h_{0}}{l_{1}^{2}-1 / 4} \\
f_{2}\left( \pm \frac{1}{2}, l_{1} ; \pm \frac{1}{2}, l_{1}\right) & =\mp \frac{2 h_{0} l_{1}}{l_{1}^{2}-1 / 4},
\end{aligned}
$$

где $h_{0}$ - произвольное число. Такой оператор $\Gamma^{\mu \nu}$ может быть выражен через описанные формулами (27) и (28) 4-векторные операторы $\Gamma^{\mu}$ и $L^{\mu}$ :

$$
\Gamma^{\mu \nu}=b_{0}^{-2} h_{0}\left(L^{\mu} L^{\nu}-L^{\nu} L^{\mu}\right)
$$

или

$$
\Gamma^{\mu \nu}=i b_{0}^{-1} c_{0}^{-1} h_{0} \varepsilon^{\mu \nu \rho \sigma} \Gamma_{\rho} L_{\sigma} .
$$

Нахождение общей структуры антисимметричных по двум индексам матричных тензорных операторов третьего и последующих рангов $\Gamma^{\mu \nu \nu_{1} \ldots \nu_{j}}, j=1,2, \ldots$, приводящих к полярному 4-вектору тока (5), является достаточно трудоемкой, но решаемой задачей. Знание общей структуры позволило бы найти все наборы констант таких операторов, при которых ток (5), (6) обладает инвариантностью относительно преобразований вторичной симметрии (4). В отсутствие соответствующих знаний мы обеспечиваем двойную инвариантность теории, вовлекая в рассмотрение только те операторы высоких рангов $\Gamma^{\mu \nu \nu_{1} \ldots \nu_{j}}$, которые выражаются через 4-векторные операторы $\Gamma^{\mu}(27)$ и $L^{\mu}(28)$. Вполне возможно, что в результате исчерпывается весь список допустимых операторов $\Gamma^{\mu \nu \nu_{1} \ldots \nu_{j}}$, но доказательства этого мы не имеем.

Требуемая нами бесследность матричного оператора $\Gamma^{\mu \nu \nu_{1} \ldots \nu_{j}}$ ранга $2+j$ означает, что этот оператор не содержит в себе матричных тензорных операторов меньшего ранга $j$. При выражении бесследных операторов $\Gamma^{\mu \nu \nu_{1} \ldots \nu_{j}}$ через операторы $\Gamma^{\mu}$ и $L^{\mu}$ необходимо принимать во внимание следующие соотношения:

$$
\begin{aligned}
\Gamma^{\mu} \Gamma_{\mu}=4 c_{0}^{2}, \quad L^{\mu} L_{\mu} & =-3 b_{0}^{2}, \quad L^{\mu} \Gamma_{\mu}=0, \\
4 b_{0}^{-2}\left(L^{\mu} L^{\nu}+L^{\nu} L^{\mu}\right)+6 g^{\mu \nu} & =c_{0}^{-2}\left(\Gamma^{\mu} \Gamma^{\nu}+\Gamma^{\nu} \Gamma^{\mu}\right)-2 g^{\mu \nu} .
\end{aligned}
$$


Последнее равенство отражает совпадение (с точностью до числового множителя) бесследных симметричных тензорных операторов второго ранга, построенных из операторов $L^{\mu}$ и из операторов $\Gamma^{\mu}$.

Перечислим теперь все линейно независимые матричные операторы третьего и четвертого рангов с требуемыми свойствами. Имеем

$$
\begin{aligned}
\Gamma_{1}^{\mu \nu \rho}= & h_{1} \Gamma^{\rho} \Gamma^{\mu \nu}, \\
\Gamma_{2}^{\mu \nu \rho}= & h_{2}\left[L^{\rho} \widetilde{\Gamma}^{\mu \nu}-\frac{b_{0} h_{0}}{c_{0}}\left(g^{\mu \rho} \Gamma^{\nu}-g^{\nu \rho} \Gamma^{\mu}\right)\right], \\
\Gamma_{1}^{\mu \nu \rho \sigma}= & h_{3}\left[\Gamma^{\rho} \Gamma^{\sigma} \Gamma^{\mu \nu}-\frac{c_{0}^{2}}{3}\left(4 g^{\rho \sigma} \Gamma^{\mu \nu}+g^{\mu \rho} \Gamma^{\nu \sigma}-g^{\nu \rho} \Gamma^{\mu \sigma}+g^{\mu \sigma} \Gamma^{\nu \rho}-g^{\nu \sigma} \Gamma^{\mu \rho}\right)\right], \\
\Gamma_{2}^{\mu \nu \rho \sigma}= & h_{4}\left[L^{\rho} \Gamma^{\sigma} \widetilde{\Gamma}^{\mu \nu}-\frac{b_{0} h_{0}}{c_{0}}\left(g^{\mu \rho} \Gamma^{\nu} \Gamma^{\sigma}-g^{\nu \rho} \Gamma^{\mu} \Gamma^{\sigma}\right)+\right. \\
& \left.+\frac{c_{0} b_{0}}{6}\left(2 g^{\rho \sigma} \Gamma^{\mu \nu}-g^{\mu \rho} \Gamma^{\nu \sigma}+g^{\nu \rho} \Gamma^{\mu \sigma}+5 g^{\mu \sigma} \Gamma^{\nu \rho}-5 g^{\nu \sigma} \Gamma^{\mu \rho}\right)\right],
\end{aligned}
$$

где

$$
\widetilde{\Gamma}^{\mu \nu}=\frac{i}{2} \varepsilon^{\mu \nu \rho \sigma} \Gamma_{\rho \sigma}=\frac{h_{0}}{b_{0} c_{0}}\left(\Gamma^{\mu} L^{\nu}-\Gamma^{\nu} L^{\mu}\right),
$$

a $h_{1}, h_{2}, h_{3}$ и $h_{4}-$ произвольные константы.

\section{5. КОНЕЧНЫЕ СОБСТВЕННО ЛОРЕНЦЕВЫ ПРЕОБРАЗОВАНИЯ ДЛЯ КОНЕЧНОМЕРНЫХ НЕПРИВОДИМЫХ ПРЕДСТАВЛЕНИЙ $\left( \pm 1 / 2, L_{1}\right)$}

Рассмотрим некоторые следствия собственно лоренцевого преобразования, отвечающего переходу от системы покоя частицы к лабораторной системе, в которой частица движется вдоль третьей координатной оси со скоростью $v$. Соответствующие преобразования в пространстве волновых векторов $\psi$ даются оператором $S(\alpha)=e^{\alpha I^{03}}$, где th $\alpha=v$. Так как инфинитезимальный оператор $I^{03}$, действуя на тот или иной вектор канонического базиса, не изменяет его проекции спина на третью ось, матричные элементы оператора $I^{03}$ и матричные элементы конечных собственно лоренцевых преобразований в пространстве любого неприводимого представления $\left(l_{0}, l_{1}\right)$ группы $L_{+}^{\uparrow}$ определяются следующими соотношениями:

$$
\begin{aligned}
I^{03} \xi_{\left(l_{0}, l_{1}\right) l m} & =\sum_{l^{\prime}} I_{l^{\prime} m, l m}^{\left(l_{0}, l_{1}\right)} \xi_{\left(l_{0}, l_{1}\right) l^{\prime} m}, \\
e^{\alpha I^{03}} \xi_{\left(l_{0}, l_{1}\right) l m} & =\sum_{l^{\prime}} A_{l^{\prime} m, l m}^{\left(l_{0}, l_{1}\right)}(\alpha) \xi_{\left(l_{0}, l_{1}\right) l^{\prime} m} .
\end{aligned}
$$

Установим ряд связей между величинами $A_{l^{\prime} m, l m}^{\left(l_{0}, l_{1}\right)}(\alpha)$, ограничиваясь конечномерными неприводимыми представлениями группы $L_{+}^{\uparrow}$. Из явного вида коэффициентов разложения (36) для таких представлений (см. работы [7]-[9]) следует, что

$$
I_{l^{\prime}-m, l-m}^{\left(l_{0}, l_{1}\right)}=I_{l^{\prime} m, l m}^{\left(-l_{0}, l_{1}\right)}=-\left[I_{l^{\prime} m, l m}^{\left(l_{0}, l_{1}\right)}\right]^{*} .
$$


Отсюда и из соотношений $(36),(37)$ получаем

$$
A_{l^{\prime}-m, l-m}^{\left(l_{0}, l_{1}\right)}(\alpha)=A_{l^{\prime} m, l m}^{\left(-l_{0}, l_{1}\right)}(\alpha)=\left[A_{l^{\prime} m, l m}^{\left(l_{0}, l_{1}\right)}(-\alpha)\right]^{*} .
$$

Отметим, что в системе покоя частицы со спином $1 / 2$ двум ее независимым состояниям, подчиняющимся уравнению (7), соответствуют волновые векторы вида $[10]$

$$
\psi_{m}\left(p_{0}\right)=\sum_{N=1}^{+\infty} \chi(N)\left[\xi_{\left(\frac{1}{2}, \frac{1}{2}+N\right) \frac{1}{2} m}+r \xi_{\left(-\frac{1}{2}, \frac{1}{2}+N\right) \frac{1}{2} m}\right],
$$

где $m=-1 / 2,1 / 2$, а $r$ описывает пространственную четность состояния и равно +1 или -1 . В системе отсчета, в которой скорость рассматриваемой частицы направлена вдоль третьей оси, волновой вектор частицы дается формулой

$$
\begin{aligned}
& \psi_{m}(p)=S(\alpha) \psi_{m}\left(p_{0}\right)= \\
& \quad=\sum_{N=1}^{+\infty} \sum_{l=1 / 2}^{N-1 / 2} \chi(N)\left[A_{l m, \frac{1}{2} m}^{\left(\frac{1}{2}, \frac{1}{2}+N\right)}(\alpha) \xi_{\left(\frac{1}{2}, \frac{1}{2}+N\right) \frac{1}{2} m}+r A_{l m, \frac{1}{2} m}^{\left(-\frac{1}{2}, \frac{1}{2}+N\right)}(\alpha) \xi_{\left(-\frac{1}{2}, \frac{1}{2}+N\right) \frac{1}{2} m}\right] .
\end{aligned}
$$

Отсюда, учитывая соотношения (39), приходим к заключению, что для вычисления формфакторов $(8),(9)$ нужно знать только величины $A_{l \frac{1}{2}, \frac{1}{2} \frac{1}{2}}^{\left(\frac{1}{2}, l_{1}\right)}(\alpha)$, причем только при $l=1 / 2$ и $3 / 2$, если ограничиваться в формуле (6) тензорными операторами второго, третьего и четвертого рангов.

Взяв производную по $\alpha$ от обеих частей равенства (37) и воспользовавшись известным видом оператора $I^{03}$ в каноническом базисе (см. работы [7]-[9]), получаем

$$
\begin{gathered}
\frac{d}{d \alpha} A_{l \frac{1}{2}, \frac{1}{2} \frac{1}{2}}^{\left(\frac{1}{2}, l_{1}\right)}(\alpha)=-\frac{i}{4(l+1)} \sqrt{(2 l+1)(2 l+3)\left(l_{1}^{2}-(l+1)^{2}\right)} A_{l+1 \frac{1}{2}, \frac{1}{2} \frac{1}{2}}^{\left(\frac{1}{2}, l_{1}\right)}(\alpha)+ \\
+\frac{l_{1}}{l(l+1)} A_{l \frac{1}{2}, \frac{1}{2} \frac{1}{2}}^{\left(\frac{1}{2}, l_{1}\right)}(\alpha)+\frac{i}{4 l} \sqrt{(2 l-1)(2 l+1)\left(l_{1}^{2}-l^{2}\right)} A_{l-1 \frac{1}{2}, \frac{1}{2} \frac{1}{2}}^{\left(\frac{1}{2}, l_{1}\right)}(\alpha) .
\end{gathered}
$$

При заданном значении числа $l_{1}$ данное рекуррентное соотношение позволяет, зная один лишь матричный элемент $A_{\frac{1}{2} \frac{1}{2}, \frac{1}{2} \frac{1}{2}}^{\left(\frac{1}{2}, l_{1}\right)}(\alpha)$ как функцию переменной $\alpha$, найти все остальные элементы вида $A_{l \frac{1}{2}, \frac{1}{2} \frac{1}{2}}^{\left(\frac{1}{2}, l_{1}\right)}(\alpha)\left(\right.$ при $\left.l=3 / 2, \ldots,\left|l_{1}\right|-1\right)$.

Приступим к выводу рекуррентного соотношения, включающего в себя величины $A_{l \frac{1}{2}, \frac{1}{2} \frac{1}{2}}^{\left(\frac{1}{2}, l_{1}\right)}(\alpha)$ с различными значениями числа $l_{1}$. Возьмем в пространстве представления $\left(1 / 2, l_{1}\right) \oplus\left(1 / 2, l_{1}+1\right)$ собственной группы Лоренца два вектора $\varphi(p)$ и $\Phi(p)$, которые в произвольной инерциальной системе отсчета, где 4-импульс рассматриваемой нами частицы равен $p$, связаны друг с другом релятивистски-ковариантным равенством

$$
\Phi(p)=\left(L^{\mu} p_{\mu}\right) \varphi(p)
$$

а в системе покоя частицы имеют в каноническом базисе только по одной ненулевой компоненте:

$$
\varphi\left(p_{0}\right)=\varphi_{0} \xi_{\left(\frac{1}{2}, l_{1}\right) \frac{1}{2} \frac{1}{2}}, \quad \Phi\left(p_{0}\right)=\Phi_{0} \xi_{\left(\frac{1}{2}, l_{1}+1\right) \frac{1}{2} \frac{1}{2}}
$$


где $\varphi_{0}$ и $\Phi_{0}-$ произвольные константы. Полное соответствие между общей структурой 4-векторного оператора, равенством (43) в системе покоя частицы, имеющим вид

$$
\Phi\left(p_{0}\right)=\left(L^{0} M\right) \varphi\left(p_{0}\right),
$$

и соотношениями (44) достигается при условиях

$$
\begin{aligned}
L^{0} \xi_{\left(\frac{1}{2}, l_{1}\right) \frac{1}{2} \frac{1}{2}} & =i a_{0} \sqrt{\left(l_{1}-1 / 2\right)\left(l_{1}+3 / 2\right)} \xi_{\left(\frac{1}{2}, l_{1}+1\right) \frac{1}{2} \frac{1}{2}} \\
\Phi_{0} & =i \varphi_{0} a_{0} M \sqrt{\left(l_{1}-1 / 2\right)\left(l_{1}+3 / 2\right)} .
\end{aligned}
$$

В лабораторной системе отсчета, характеризуемой параметром $\alpha$ лоренцевого буста, из равенства (43) с учетом формул (37) и (44) следует, что

$$
\sum_{l} \Phi_{0} A_{l \frac{1}{2}, \frac{1}{2} \frac{1}{2}}^{\left(\frac{1}{2}, l_{1}+1\right)}(\alpha) \xi_{\left(\frac{1}{2}, l_{1}+1\right) l \frac{1}{2}}=\left(L^{0} M \operatorname{ch} \alpha-L^{3} M \operatorname{sh} \alpha\right) \sum_{l^{\prime}} \phi_{0} A_{l^{\prime} \frac{1}{2}, \frac{1}{2} \frac{1}{2}}^{\left(\frac{1}{2}, l_{1}\right)}(\alpha) \xi_{\left(\frac{1}{2}, l_{1}\right) l^{\prime} \frac{1}{2}} .
$$

Учитывая, что $L^{3}=\left[L^{0}, I^{03}\right]$ (см. [7]-[9]), принимая во внимание явный вид оператора $I^{03}$ в каноническом базисе и формулы (46), получаем из (47) следующее рекуррентное соотношение:

$$
\begin{aligned}
A_{l \frac{1}{2}, \frac{1}{2} \frac{1}{2}}^{\left(\frac{1}{2}, l_{1}+1\right)}(\alpha)= & \frac{1}{2 l(l+1) \sqrt{\left(2 l_{1}-1\right)\left(2 l_{1}+3\right)}} \times \\
& \times\left[-i l \operatorname{sh} \alpha \sqrt{(2 l+1)(2 l+3)\left(l_{1}-l-1\right)\left(l_{1}-l\right)} A_{l+\frac{1}{2}, \frac{1}{2} \frac{1}{2}}^{\left(\frac{1}{2}, l_{1}\right)}(\alpha)+\right. \\
& +(4 l(l+1) \operatorname{ch} \alpha+\operatorname{sh} \alpha) \sqrt{\left(l_{1}-l\right)\left(l_{1}+l+1\right)} A_{l \frac{1}{2}, \frac{1}{2} \frac{1}{2}}^{\left(\frac{1}{2}, l_{1}\right)}(\alpha)+ \\
& \left.+i(l+1) \operatorname{sh} \alpha \sqrt{(2 l-1)(2 l+1)\left(l_{1}+l\right)\left(l_{1}+l+1\right)} A_{l-1 \frac{1}{2}, \frac{1}{2} \frac{1}{2}}^{\left(\frac{1}{2}, l_{1}\right)}(\alpha)\right]
\end{aligned}
$$

где $l_{1}=3 / 2,5 / 2, \ldots$, a $l=1 / 2, \ldots, l_{1}$.

Теперь, пользуясь методом математической индукции, мы в состоянии доказать, что справедливо равенство

$$
A_{\frac{1}{2} \frac{1}{2}, \frac{1}{2} \frac{1}{2}}^{\left(\frac{1}{2}, l_{1}\right)}(\alpha)=\frac{2}{l_{1}^{2}-1 / 4} \sum_{n=0}^{l_{1}-3 / 2}\left(l_{1}-n-\frac{1}{2}\right) e^{\left(l_{1}-2 n-1\right) \alpha},
$$

где $l_{1}=3 / 2,5 / 2, \ldots$ В первую очередь мы убеждаемся в справедливости формулы (49) при $l_{1}=3 / 2$, когда она принимает вид

$$
A_{\frac{1}{2} \frac{1}{2}, \frac{1}{2} \frac{1}{2}}^{\left(\frac{1}{2}, \frac{3}{2}\right)}(\alpha)=e^{\alpha / 2}
$$

Для этого достаточно учесть известное равенство [7]-[9]

$$
I^{03} \xi_{\left(\frac{1}{2}, \frac{3}{2}\right) \frac{1}{2} \frac{1}{2}}=\frac{1}{2} \xi_{\left(\frac{1}{2}, \frac{3}{2}\right) \frac{1}{2} \frac{1}{2}}
$$

и соотношение (37). Предполагая справедливость формулы (49) для некоторого значения числа $l_{1}$, равного $k_{1}, k_{1}=5 / 2,7 / 2, \ldots$, имеем на основании (42), что

$$
A_{\frac{3}{2} \frac{1}{2}, \frac{1}{2} \frac{1}{2}}^{\left(\frac{1}{2}, k_{1}\right)}(\alpha)=\frac{2 i \sqrt{2}}{\left(k_{1}^{2}-1 / 4\right) \sqrt{k_{1}^{2}-9 / 4}} \sum_{n=0}^{k_{1}-3 / 2}\left(k_{1}-n-\frac{1}{2}\right)\left(k_{1}-3 n-\frac{3}{2}\right) e^{\left(k_{1}-2 n-1\right) \alpha} .
$$


Пользуясь теперь рекуррентным соотношением (48), находим, что получаемая из него величина $A_{\frac{1}{2} \frac{1}{2}, \frac{1}{2} \frac{1}{2}}^{\left(\frac{1}{2}, k_{1}+1\right)}(\alpha)$ равна правой части равенства $(49)$ при замене в нем числа $l_{1}$ на $k_{1}+1$, т. е. что формула (49) справедлива и для значения числа $l_{1}$, равного $k_{1}+1$.

\section{6. ЗАПИСЬ КОМПОНЕНТ ПОЛЕВОГО ВЕКТОРА В ВИДЕ НЕПРЕРЫВНЫХ ДРОБЕЙ}

Займемся вопросом вычисления полевого вектора $\psi(p)$, принадлежащего пространству представления $S^{3 / 2}(26)$ группы $L_{+}^{\uparrow}$ и удовлетворяющего уравнению (7), в котором оператор $\Gamma^{0}$ дается соотношением (27), а оператор $R$ имеет вид [13], [14]

$$
R \xi_{\left( \pm \frac{1}{2}, \frac{1}{2}+N\right) l m}=\rho(N) \xi_{\left( \pm \frac{1}{2}, \frac{1}{2}+N\right) l m}=\left[\sum_{i} \lambda_{i} \eta_{i}(N)\right] \xi_{\left( \pm \frac{1}{2}, \frac{1}{2}+N\right) l m}
$$

причем

$$
\eta_{i}(N)=2 \frac{v_{i}^{N}\left(v_{i} N+N+1\right)-w_{i}^{N}\left(w_{i} N+N+1\right)}{N(N+1)\left(v_{i}-w_{i}\right)\left(2+v_{i}+w_{i}\right)},
$$

где $N=1,2, \ldots, v_{i}=\left(z_{i}+\sqrt{z_{i}^{2}-4}\right) / 2, w_{i}=\left(z_{i}-\sqrt{z_{i}^{2}-4}\right) / 2, \lambda_{i}$ и $z_{i}$ - некоторые действительные константы, а индекс $i$ принимает одно или несколько значений.

Введем систему отсчета, в которой 4-импульс равен $p_{0}=\left\{M_{0}, 0,0,0\right\}$, и рассмотрим состояние поля $\psi\left(p_{0}\right)$, обладающее в этой системе спином $1 / 2$ и описываемое равенством (40). Тогда уравнение (7) сводится к следующему рекуррентному соотношению относительно компонент полевого вектора $\chi(N)$ :

$$
\begin{aligned}
N \sqrt{N(N+2)} \chi(N+1) & +(N+1) \sqrt{(N-1)(N+1)} \chi(N-1)+ \\
+ & {\left[\left(M_{0} c_{0}\right)^{-1} N(N+1) \rho(N)-r\right] \chi(N)=0, }
\end{aligned}
$$

где $N=1,2, \ldots$ и к тому же $\chi(0)=0$.

Соотношение (55) позволяет при любых значениях свободных параметров рассматриваемой теории выразить все величины $\chi(N), N=2,3, \ldots$, через одну величину $\chi(1)$. Для физики частиц представляют интерес лишь те совокупности свободных параметров теории, при которых получаемые величины $\chi(N)$ обеспечивают нормируемость соответствующих полевых векторов $\psi\left(p_{0}\right)(40)$. Выполнение этого требования при том или ином фиксированном наборе свободных параметров $z_{i}, \lambda_{i}$ и $c_{0}$ приводит к выделению некоторого множества значений параметра $M_{0}$, приобретающих статус масс частиц со спином покоя $1 / 2$ и пространственной четностью $r$.

Подробный анализ характеристик спектра масс теории в зависимости от области, которой принадлежат свободные параметры $z_{i}$, изложен в работе [14]. Показано, что экспериментальная картина нуклонных резонансов удовлетворительно согласуется со спектром масс изучаемой теории полей класса ISFIR, если положить в формулах (53) и (54) $z_{1}>2,\left|z_{j}\right|<2$ при $j \neq 1$. В этом случае, который мы будем рассматривать в дальнейшем, асимптотика компонент полевых векторов задается только параметром $z_{1}$ : при $N \rightarrow \infty$ имеем

$$
\chi(N)=A_{0} G(N)\left(1+O\left(N^{-1}\right)\right)+B_{0} G^{-1}(N)\left(1+O\left(N^{-1}\right)\right),
$$



где

$$
G(N)=\left(-\frac{\rho(1)}{M_{0} c_{0}}\right)^{N} \frac{v_{1}^{N(N+1) / 2}}{N !} .
$$

Нормируемость полевого вектора $\psi\left(p_{0}\right)(40)$ обеспечивается тогда и только тогда, когда коэффициент $A_{0}$ из соотношения (56), зависящий от свободных параметров теории $M_{0}, c_{0}, \lambda_{i}$, обращается в нуль. Мы не располагаем никаким аналитическим методом решения уравнения $A_{0}=0$ относительно параметра $M_{0}$, т. е. методом нахождения спектра масс, присущего уравнению (55). Задача расчета любого количества низших значений масс решается приближенно численными методами, алгоритм которых описан в работе [14]. Однако всякое приближение к значению массы приводит хотя и к малой, но ненулевой величине $A_{0}$, в результате чего находимые из точного соотношения (55) величи́ны $\chi(N)$ уже при небольших номерах $N$ переходят от убывающих с ростом $N$ значений к быстро растущим. (Так, при значениях параметров теории, приведенных в начале раздела 8 , рост последовательности $\chi(N)$ начинается с восьмого члена.) Это существенно сужает возможности использования численных расчетов.

Ниже мы представим формулу для вычисления величин $\chi(N)$, обладающую следующими свойствами. Она сама по себе является приближенным соотношением для величин $\chi(N)$, если параметр $M_{0}$ отличается от массы состояния $M$, и точным соотношением, если $M_{0}=M$. Получаемые из этой формулы значения $\chi(N)$ стремятся к нулю при бесконечном росте $N$.

Введем обозначения

$$
\pi(N)=(-1)^{N} \sqrt{N(N+1)} \chi(N), \quad H(N)=\left(M_{0} c_{0}\right)^{-1} N(N+1) \rho(N)-r .
$$

Тогда соотношение (55) перепишется в виде

$$
N^{2} \pi(N+1)+(N+1)^{2} \pi(N-1)-H(N) \pi(N)=0,
$$

где $N=1,2, \ldots$. Эту систему следует дополнить условием $\pi(0)=0$ или эквивалентным ему условием

$$
\pi(2)=H(1) \pi(1)
$$

получаемым из (59) при $N=1$. Уравнения (59) с учетом равенства (60) позволяют выразить все искомые величины $\pi(N)$ через $\pi(1)$. Имеем

$$
\frac{\pi(N)}{\pi(N+1)}=\frac{N^{2}}{H(N)-\frac{(N-1)^{2}(N+1)^{2}}{H(N-1)-\frac{(N-2)^{2} N^{2}}{\ddots \cdot}}},
$$

где $N=2,3, \ldots$.

Допустим теперь, что выполнение условия $\pi(0)=0$ не является обязательным дополнением к соотношению (59). Тем самым мы исключим из рассмотрения одно 
из уравнений (59), в котором $N=1$. Остающиеся уравнения системы (59) (при $N=2,3, \ldots)$ имеют два независимых решения, так как все величины $\pi(N)$ можно выразить через $\pi(1)$ и $\pi(2)$. Одно из этих решений дается выражениями (60), (61), а другое можно представить в виде бесконечной непрерывной дроби:

$$
\frac{\pi(N+1)}{\pi(N)}=\frac{(N+2)^{2}}{H(N+1)-\frac{(N+1)^{2}(N+3)^{2}}{(N+2)^{2}(N+4)^{2}}},
$$

где $N=1,2, \ldots$.

Бесконечную непрерывную дробь (62) следует рассматривать как предел последовательности

$$
\begin{aligned}
& {\left[\frac{\pi(N+1)}{\pi(N)}\right]_{1}=\frac{(N+2)^{2}}{H(N+1)},} \\
& {\left[\frac{\pi(N+1)}{\pi(N)}\right]_{2}=\frac{(N+2)^{2}}{H(N+1)-\frac{(N+1)^{2}(N+3)^{2}}{H(N+2)}}, \ldots,}
\end{aligned}
$$

которая возникает в результате решения системы уравнений (59) с номерами $N=$ $2,3, \ldots$ методом последовательных приближений:

$$
N^{2}\left[\frac{\pi(N+1)}{\pi(N)}\right]_{n-1}+(N+1)^{2}\left\{\left[\frac{\pi(N)}{\pi(N-1)}\right]_{n}\right\}^{-1}-H(N)=0,
$$

где $n=1,2, \ldots$, причем $[\pi(N+1) / \pi(N)]_{0}=0$.

Докажем, что в рассматриваемой нами области параметров $z_{i}$ из формул (53) и (54) последовательность $[\pi(N+1) / \pi(N)]_{n}$ сходится при $n \rightarrow \infty$. Для этого учтем, что величина $\eta_{1}(N)$ растет экспоненциально с ростом $N$, а значения величины $\eta_{j}(N)$, $j \neq 1$, при изменении $N$ осциллируют в ограниченной области. Поэтому при достаточно больших значениях числа $N$ вклад величин $\eta_{j}(N), j \neq 1$, в $\rho(N)$, а затем в $H(N)$ становится сколь угодно малым. Тем самым для любого набора свободных параметров теории $M_{0}, c_{0}, \lambda^{i}$ и для любого числа $\varepsilon>0$ можно указать такой зависящий от них номер $N_{0}$, что для всех $N \geqslant N_{0}$ справедливо неравенство (ради простоты будем полагать далее, что $\left.M_{0} c_{0} \rho\left(N_{0}\right)>0\right) 0<N^{4} / H(N)<\varepsilon$ и, следовательно, неравенство

$$
0<\frac{(N+1)^{2}(N+2)^{2}}{H(N+1)}<\frac{(2 N-1) H(N)}{N^{2}} .
$$

С помощью этого соотношения легко установить, что при $N \geqslant N_{0}$ последовательность $[\pi(N+1) / \pi(N)]_{n}, n=1,2, \ldots$, является монотонно растущей, ограниченной 

сверxy,

$$
0<\left[\frac{\pi(N+1)}{\pi(N)}\right]_{n}<\frac{(N+1)^{2}(N+2)^{2}}{N^{2} H(N+1)},
$$

и поэтому сходящейся. Вслед за этим на основании соотношения (64) устанавливается сходимость последовательности $[\pi(N+1) / \pi(N)]_{n}, n=1,2, \ldots$, поочередно для $N=N_{0}-1, N_{0}-2, \ldots, 1$.

Переходя в неравенствах (66) вначале к пределу $n \rightarrow \infty$, а затем к пределу $N \rightarrow \infty$, получаем

$$
\lim _{N \rightarrow \infty} \frac{\pi(N+1)}{\pi(N)}=0, \quad \lim _{N \rightarrow \infty} \pi(N)=0 .
$$

Отсюда следует, что при любом значении параметра $M_{0}$ компоненты полевого вектора $\chi(N)$, находимые с помощью формул $(58),(62)$, стремятся к нулю при бесконечном росте $N$.

Если правую часть равенства (62) при $N=1$ приравнять к $H(1)$, то полученное уравнение при некотором фиксированном значении параметров $z_{i}, \lambda_{i}$ и $c_{0}$ можно рассматривать как уравнение на спектр масс состояний со спином покоя $1 / 2$ и четностью $r$. При выполнении указанного уравнения величины $\pi(N)$, получаемые с помощью формулы (62), удовлетворяют соотношению (59) при всех требуемых значениях $N$, а соответствующие полевые векторы $\psi\left(p_{0}\right)$ (40) являются нормируемыми. При малом отклонении значения параметра $M_{0}$ от некоторой точки $M$ спектра масс приближенные величины $\pi(N)_{\left.\right|_{M_{0}}}$, рассчитанные по формуле (62), и точные величины $\pi(N)_{\left.\right|_{\mathrm{M}}}$, удовлетворяющие как соотношению (62), так и равенству (60), имеют схожую асимптотику при росте $N$ и мало отличаются друг от друга. Степень той и другой малости можно оценить при выполнении подходящих численных расчетов.

\section{7. НЕКОТОРЫЕ АСПЕКТЫ АНАЛИТИЧЕСКИХ ВЫЧИСЛЕНИЙ ВКЛАДОВ В ФОРМФАКТОРЫ ТЕНЗОРНЫХ ОПЕРАТОРОВ НИЗШИХ РАНГОВ}

Окончательные аналитические выражения для электромагнитных формфакторов (8), (9) даже при вычислении вкладов только тензорных операторов низших (до четвертого) рангов достаточно громоздкие. Поэтому ограничимся изложением ряда существенных элементов, которые прямо ведут к таким выражениям.

Отметим вначале, что без ограничения общности пространственную четность $r$ рассматриваемого состояния, входящую в соотношения (40), (41), (55) и (58), можно положить равной единице.

Билинейная форма $\left(\psi_{1}, \psi_{2}\right)$ в пространстве представления группы $L_{+}^{\uparrow}$, разложимого в прямую сумму конечномерных неприводимых представлений, является релятивистски-инвариантной, если [7], [8]

$$
\left(\xi_{\tau^{\prime} l^{\prime} m^{\prime}}, \xi_{\tau l m}\right)=(-1)^{[l]} \delta_{\tau^{\prime} \tau^{*}} \delta_{l^{\prime} l} \delta_{m^{\prime} m}
$$

где $\tau^{*}=\left(l_{0},-l_{1}\right) \sim\left(-l_{0}, l_{1}\right)$ при $\tau=\left(l_{0}, l_{1}\right)$. 
Чтобы обеспечить вещественность формфакторов (8), будем полагать, что константы $b_{0}$ и $h_{i}, i=0,1,2,3,4$, в формулах (28)-(35) действительны. Тогда

$$
\left(\psi_{1}, \Gamma^{\mu} \psi_{2}\right)=\left(\Gamma^{\mu} \psi_{1}, \psi_{2}\right), \quad\left(\psi_{1}, L^{\mu} \psi_{2}\right)=\left(L^{\mu} \psi_{1}, \psi_{2}\right), \quad\left(\psi_{1}, \Gamma^{\mu \nu} \psi_{2}\right)=-\left(\Gamma^{\mu \nu} \psi_{1}, \psi_{2}\right) .
$$

Отсюда получаем равенства

$$
\begin{aligned}
2\left(\psi_{1}, \Gamma^{\rho} \Gamma^{\mu \nu} \psi_{2}\right) & =\left(\Gamma^{\rho} \psi_{1}, \Gamma^{\mu \nu} \psi_{2}\right), & \left(\psi_{1}, L^{\rho} \widetilde{\Gamma}^{\mu \nu} \psi_{2}\right) & =\left(L^{\rho} \psi_{1}, \widetilde{\Gamma}^{\mu \nu} \psi_{2}\right), \\
\left(\psi_{1}, \Gamma^{\rho} \Gamma^{\sigma} \Gamma^{\mu \nu} \psi_{2}\right) & =\left(\Gamma^{\sigma} \Gamma^{\rho} \psi_{1}, \Gamma^{\mu \nu} \psi_{2}\right), & \left(\psi_{1}, L^{\rho} \Gamma^{\sigma} \widetilde{\Gamma}^{\mu \nu} \psi_{2}\right) & =\left(\Gamma^{\sigma} L^{\rho} \psi_{1}, \widetilde{\Gamma}^{\mu \nu} \psi_{2}\right),
\end{aligned}
$$

которые значительно облегчают и аналитические, и численные расчеты.

Нам удалось обнаружить ряд соотношений, которым удовлетворяют отдельные члены формфакторов (8), (9), а именно

$$
\begin{aligned}
\left(\psi_{\frac{1}{2}}(p), \Gamma_{i}^{\mu \nu \rho} q_{\nu} q_{\rho} \psi_{m}\left(p_{0}\right)\right) & =0 \\
\left(\psi_{\frac{1}{2}}(p), \Gamma_{i}^{\mu \nu \rho \sigma} q_{\nu} p_{\rho} q_{\sigma} \psi_{m}\left(p_{0}\right)\right) & =-\frac{1}{2}\left(\psi_{\frac{1}{2}}(p), \Gamma_{i}^{\mu \nu \rho \sigma} q_{\nu} q_{\rho} q_{\sigma} \psi_{m}\left(p_{0}\right)\right),
\end{aligned}
$$

где $\mu=0,1$, a $i=1,2$.

Доказательства формул (71), (72) опираются на трансформационные свойства относительно лоренцевого буста вдоль третьей оси 4-векторных матричных операторов $K^{\mu}[7]-[9]$ и антисимметричных тензорных операторов $K^{\mu \nu}(14)$,

$$
K^{\mu} p_{\mu} S(\alpha)=S(\alpha) K^{\mu} p_{0 \mu}, \quad K^{03} S(\alpha)=S(\alpha) K^{03}, \quad K^{1 \mu} p_{\mu} S(\alpha)=S(\alpha) K^{1 \mu} p_{0 \mu},
$$

а также используют равенства (69), заданность $P$-четности состояния частицы в системе покоя, релятивистскую инвариантность билинейной формы и ее вещественность. Продемонстрируем это в самом простом случае, когда рассматривается формула (71) при $i=1$ и $\mu=0$ : имеем

$$
\begin{aligned}
\left(\psi_{\frac{1}{2}}(p),\right. & \left.\Gamma^{\rho} q_{\rho} \Gamma^{03} q_{\nu} \psi_{\frac{1}{2}}\left(p_{0}\right)\right)=\left(\left(\Gamma^{\rho} p_{\rho}-\Gamma^{\rho} p_{0 \rho}\right) S(\alpha) \psi_{\frac{1}{2}}\left(p_{0}\right), \Gamma^{03} \psi_{\frac{1}{2}}\left(p_{0}\right)\right)= \\
& =\left(\left(S(\alpha) \Gamma^{\rho} p_{0 \rho}-\Gamma^{\rho} S(\alpha) p_{0 \rho}\right) P \psi_{\frac{1}{2}}\left(p_{0}\right), \Gamma^{03} P \psi_{\frac{1}{2}}\left(p_{0}\right)\right)= \\
& =\left(\left(S(-\alpha) \Gamma^{\rho} p_{0 \rho}-\Gamma^{\rho} S(-\alpha) p_{0 \rho}\right) \psi_{\frac{1}{2}}\left(p_{0}\right), P^{2}\left(-\Gamma^{03}\right) \psi_{\frac{1}{2}}\left(p_{0}\right)\right)= \\
& =-\left(S^{-1}(\alpha)\left(\Gamma^{\rho} p_{0 \rho}-\Gamma^{\rho} p_{\rho}\right) \psi_{\frac{1}{2}}\left(p_{0}\right), \Gamma^{03} \psi_{\frac{1}{2}}\left(p_{0}\right)\right)= \\
& =\left(\Gamma^{\rho} q_{\rho} \psi_{\frac{1}{2}}\left(p_{0}\right), \Gamma^{03} S(\alpha) \psi_{\frac{1}{2}}\left(p_{0}\right)\right)=\left(\Gamma^{03} \psi_{\frac{1}{2}}(p), \Gamma^{\rho} q_{\rho} \psi_{\frac{1}{2}}\left(p_{0}\right)\right)= \\
& =-\left(\psi_{\frac{1}{2}}(p), \Gamma^{\rho} q_{\rho} \Gamma^{03} q_{\nu} \psi_{\frac{1}{2}}\left(p_{0}\right)\right) .
\end{aligned}
$$

Принимая во внимание соотношения (33)-(35), можно показать, что

$$
\Gamma_{2}^{\mu \nu \rho \sigma} q_{\nu} q_{\rho} q_{\sigma}=c \Gamma_{1}^{\mu \nu \rho \sigma} q_{\nu} q_{\rho} q_{\sigma}
$$

где $c$ - некоторый числовой коэффициент. Тем самым в своих дальнейших вычислениях мы ограничиваемся тензором (6) следующего вида:

$$
\Lambda^{\mu \nu}\left(p, p_{0}\right)=\Gamma^{\mu \nu}+\Gamma_{1}^{\mu \nu \rho} p_{0 \rho}+\Gamma_{2}^{\mu \nu \rho} p_{0 \rho}+\Gamma_{1}^{\mu \nu \rho \sigma}\left(a_{1} q_{\rho} q_{\sigma}+a_{2} p_{0 \rho} p_{0 \sigma}\right)+\Gamma_{2}^{\mu \nu \rho \sigma} p_{0 \rho} p_{0 \sigma}
$$

где $a_{1}$ и $a_{2}-$ произвольные константы. 
Введем обозначения

$$
\xi_{l_{1}, l m}^{ \pm}=\xi_{\left(-\frac{1}{2}, l_{1}\right) l m} \pm \xi_{\left(\frac{1}{2}, l_{1}\right) l m}
$$

а также

$$
\begin{aligned}
e_{1}(N)= & \frac{\sqrt{N(N+2)}}{N+1} \chi(N+1)+\frac{2 N+1}{N(N+1)} \chi(N)- \\
& -\frac{\sqrt{(N-1)(N+1)}}{N} \chi(N-1), \\
e_{2}(N) & =\frac{\sqrt{(N-1) N}}{N+1} \chi(N+1)-\frac{\sqrt{(N-1)(N+2)}}{N(N+1)} \chi(N)- \\
& -\frac{\sqrt{(N+1)(N+2)}}{N} \chi(N-1), \\
e_{3}(N) & =\frac{\sqrt{N(N+2)}}{N+1} \chi(N+1)-\frac{N 2+N+1}{N(N+1)} \chi(N)+ \\
& +\frac{\sqrt{(N-1)(N+1)}}{N} \chi(N-1), \\
e_{4}(N) & =\frac{\sqrt{(N-1) N}}{N+1} \chi(N+1)+\frac{(2 N+1) \sqrt{(N-1)(N+2)}}{N(N+1)} \chi(N)+ \\
& +\frac{\sqrt{(N+1)(N+2)}}{N} \chi(N-1) .
\end{aligned}
$$

Получаем формулы, существенно облегчающие процедуру вычисления формфакторов (8), (9):

$$
\begin{aligned}
\Gamma^{1} \psi_{-\frac{1}{2}}\left(p_{0}\right) & =\frac{c_{0} \sqrt{2}}{6}\left(\sum_{N=1}^{+\infty} \sqrt{2} e_{1}(N) \xi_{\frac{1}{2}+N, \frac{1}{2} \frac{1}{2}}^{-}+\sum_{N=2}^{+\infty} i e_{2}(N) \xi_{\frac{1}{2}+N, \frac{3}{2} \frac{1}{2}}^{+}\right), \\
\Gamma^{03} \psi_{\frac{1}{2}}\left(p_{0}\right) & =\frac{h_{0} \sqrt{2}}{3}\left(\sum_{N=1}^{+\infty} \sqrt{2} e_{1}(N) \xi_{\frac{1}{2}+N, \frac{1}{2} \frac{1}{2}}^{-}+\sum_{N=2}^{+\infty} i e_{2}(N) \xi_{\frac{1}{2}+N, \frac{3}{2} \frac{1}{2}}^{+}\right), \\
\Gamma^{10} \psi_{-\frac{1}{2}}\left(p_{0}\right) & =-\frac{h_{0} \sqrt{2}}{6}\left(\sum_{N=1}^{+\infty} 2 \sqrt{2} e_{1}(N) \xi_{\frac{1}{2}+N, \frac{1}{2} \frac{1}{2}}^{-}-\sum_{N=2}^{+\infty} i e_{2}(N) \xi_{\frac{1}{2}+N, \frac{3}{2} \frac{1}{2}}^{+}\right), \\
\Gamma^{13} \psi_{-\frac{1}{2}}\left(p_{0}\right) & =\frac{h_{0} \sqrt{2}}{6}\left(\sum_{N=1}^{+\infty} 2 \sqrt{2} e_{3}(N) \xi_{\frac{1}{2}+N, \frac{1}{2} \frac{1}{2}}^{+}-\sum_{N=2}^{+\infty} i e_{4}(N) \xi_{\frac{1}{2}+N, \frac{3}{2} \frac{1}{2}}^{-}\right)
\end{aligned}
$$

Так как временная компонента матричного 4-векторного оператора не изменяет спин и его проекцию на третью ось, то при вычислении вкладов в формфакторы (8), (9) матричных тензоров третьего и четвертого порядков, входящих в формулу (76), удобно пользоваться соотношениями (70) и следующим равенством:

$$
\Gamma^{\sigma} \Gamma^{\rho} q_{\sigma} q_{\rho} \psi_{\frac{1}{2}}(p)=M^{2}\left[S(\alpha) \Gamma^{0} \Gamma^{0}-2 \Gamma^{0} S(\alpha) \Gamma^{0}+\Gamma^{0} \Gamma^{0} S(\alpha)\right] \psi_{\frac{1}{2}}\left(p_{0}\right) .
$$




\section{8. ПАДАЮЩИЕ С РОСТОМ $Q^{2}$ ФОРМФАКТОРЫ В ТЕОРИИ ПОЛЕЙ КЛАССА ISFIR С ЛОКАЛЬНЫМ ЭЛЕКТРОМАГНИТНЫМ ВЗАИМОДЕЙСТВИЕМ}

Те или иные суждения о возможной зависимости электромагнитных формфакторов (8), (9) от квадрата переданного импульса $Q^{2}$ в теории полей класса ISFIR являются оправданными с точки зрения физики адронов, если вначале решена задача относительно спектров масс в некоторых вариантах теории и при определенных значениях свободных параметров получено удовлетворительное согласие с экспериментальной картиной. Такое решение, допускающее удовлетворительное соответствие множеству наблюдаемых нуклонных резонансов, представлено в работе [14]. Ограничение свободы выбора в рассматриваемом нами варианте теории полей класca ISFIR состоит в следующем. Скалярный оператор $R$ (53) задается двумя параметрами $z_{1}=2.036$ и $z_{2}=0.14$, и к тому же полагаем $\lambda_{1} / c_{0}=939 \cdot 2.4686 \mathrm{MэB}$, $\lambda_{2} / \lambda_{1}=-0.6724$. Мы далее имеем дело с наинизшим по массе состоянием со спином покоя $1 / 2$.

Отметим вначале, что при приведенных значениях параметров теории магнитный момент рассматриваемой недираковской частицы, обусловленный минимальным электромагнитным взаимодействием, равен в магнетонах числу 0.2824 , а не единице, как для дираковской частицы.

Затем отметим, что при указанных значениях констант теории величи́ны $\rho(N)$ из формулы (53) для всех натуральных чисел $N$ положительны. Вследствие этого та часть электрического формфактора (8) заряженной частицы $\left(Q_{0}=1\right)$, которая порождается минимальным электромагнитным взаимодействием, $\left(\psi_{\frac{1}{2}}(p), R \psi_{\frac{1}{2}}\left(p_{0}\right)\right)$, монотонно растет до бесконечности с ростом $Q^{2}$ от 0 до $+\infty$. Устранение этого роста путем добавления в формфактор (8) слагаемого, обусловленного неминимальным электромагнитным взаимодействием, не вызывало бы никакой озабоченности, если бы подходящие значения свободных констант оператора $\Lambda^{\mu \nu}(6)$ задавались некоторой математически ясной процедурой.

В отсутствие такой процедуры мы ограничиваемся установлением принципиальной возможности такой фиксации числовых параметров оператора $\Lambda^{\mu \nu}$, при которой и формфактор (8), и формфактор (9) обеспечивают удовлетворительную аппроксимацию экспериментальных данных для протона в области небольших значений квадрата переданного импульса $\left(Q^{2} \leqslant 0.5(\text { ГэВ/c })^{2}\right)$. Поскольку оба формфактора (8) и (9) задаются одной и той же величиной (тензором $\Lambda^{\mu \nu}$ ), поэтому априори нет никаких гарантий того, что при каком-то выборе свободных констант зависимость от $Q^{2}$ и того, и другого из них будет согласована с экспериментально наблюдаемой. В справедливости этого утверждения мы убеждаемся на следующем примере. Положим, что в соотношении (6) $p_{k}=q$ для любого индекса $k$. Тогда в формфакторы (8) и (9) дают ненулевые вклады только тензоры $\Gamma^{\mu \nu \nu_{1} \ldots \nu_{j}}$ четных рангов. В результате численных расчетов с учетом вкладов тензорных операторов до шестого ранга включительно мы находим, что в состоянии обеспечить падение магнитного формфактора (9) с ростом $Q^{2}$, близкое к дипольному. Но при этом электрический формфактор (8) оказывается медленно растущим с ростом $Q^{2}$, что противоречит экспериментальной картине. 

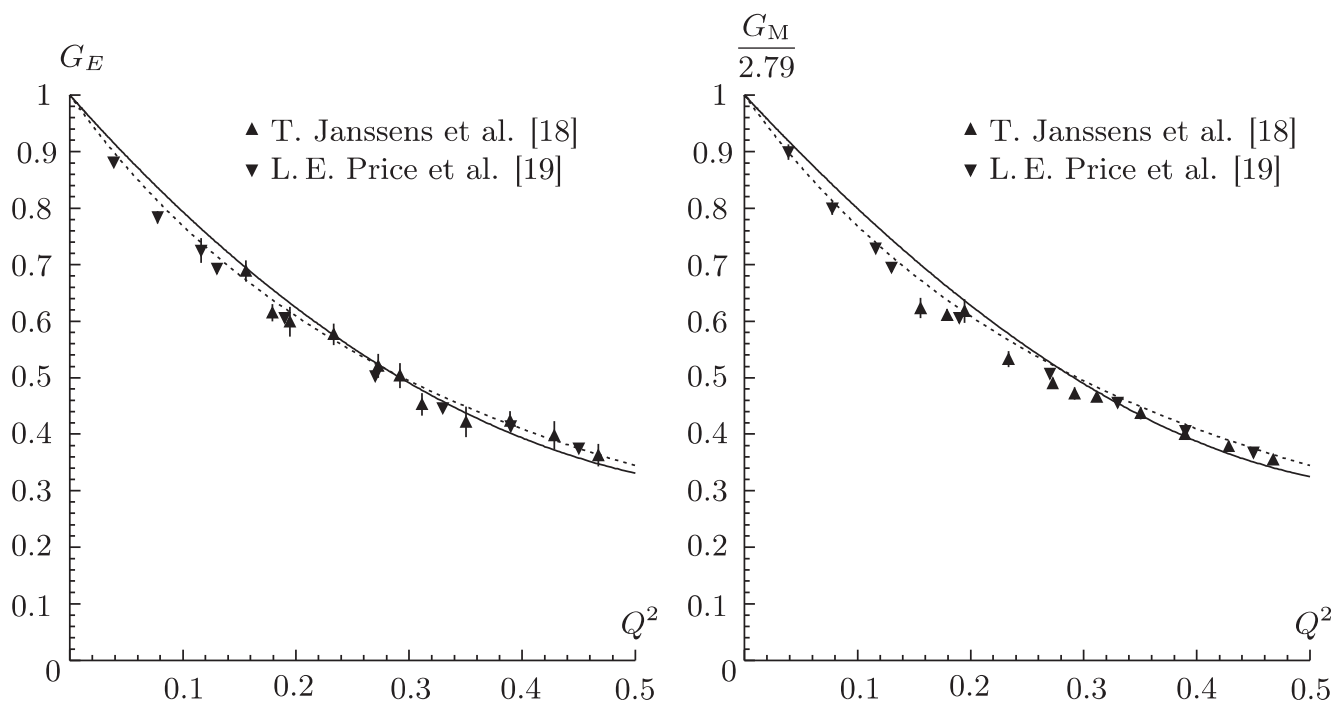

Зависимость электрического $G_{\mathrm{E}}$ и магнитного $G_{\mathrm{M}}$ формфакторов протона от квадрата переданного импульса $Q^{2}$ (в единицах $\left.(\Gamma \ni \mathrm{B} / \mathrm{c})^{2}\right)$.

Результаты аппроксимации экпериментальных данных для электрического и магнитного формфакторов протона, полученные с использованием соотношений (8)-(10), (19), (20), (27)-(31), (34), (37), (40), (49), (52)-(54), (58), (62), (68), (70), (75)-(80), показаны на рисунке сплошной линией. Они соответствуют следующему выбору констант:

$$
\begin{gathered}
\frac{M C h_{0} \sqrt{2}}{3}=6.9483, \quad c_{0} h_{1}=-1.4565, \quad b_{0} h_{2}=-0.6959, \\
a_{1}=0, \quad c_{0}^{2} h_{3} a_{2}=0.8008, \quad c_{0} b_{0} h_{4}=-0.1761 .
\end{gathered}
$$

На рисунке также изображены пунктирными линиями дипольные аппроксимации формфакторов

$$
G_{\mathrm{M}}=(1+\kappa)\left(1+\frac{Q^{2}}{0.71}\right)^{-2}, \quad G_{\mathrm{E}}=\left(1+\frac{Q^{2}}{0.71}\right)^{-2},
$$

где $Q^{2}$ дается в единицах $(\text { Гэ } / \mathrm{c})^{2}$.

\section{9. ЗАКЛЮЧИТЕЛЬНЫЕ ЗАМЕЧАНИЯ}

Полученные нами результаты в основном носят общий характер и могут быть полезными при исследовании тех или иных теорий недираковских фермионов как со спином покоя $1 / 2$, так и с более высокими спинами покоя.

Может быть, в некоторой другой публикации мы, опираясь на приведенную в разделе 3 общую структуру матричных антисимметричных операторов второго ранга, проанализируем возможность того, что оператор спина и оператор магнитного момента недираковской частицы со спином покоя $1 / 2$ существенно различны между 
собой. Это, вдобавок к уже поднятому в работе [20] вопросу о степени обоснованности формулы Баргмана-Мишеля-Телегди [21], описывающей вращение спина релятивистской частицы в постоянном однородном магнитном поле, послужило бы дополнительным аргументом в пользу необходимости экспериментальной проверки названной формулы.

Итоги той части настоящей работы, которые касаются исследуемой нами теории полей класса ISFIR, не закрывают перспективу удачного сопоставления структурным частицам локального лагранжиана их электромагнитного взаимодействия. Вместе с тем они указывают на существование нетривиальных моментов, требующих своего осмысления. Нам представляется целесообразным, используя в полной мере результаты, полученные здесь и в работах [9], [13], [14], проанализировать вариант теории полей класса ISFIR, свободные состояния которых подчиняются следующему уравнению второго порядка:

$$
\left(c_{1} \partial^{\mu} \partial_{\mu}+c_{2} \Gamma^{\mu} \Gamma^{\nu} \partial_{\mu} \partial_{\nu}+i \Gamma^{\mu} \partial_{\mu}-R\right) \Psi(x)=0
$$

с известными нам элементами (представлением группы $L_{+}^{\uparrow}(26)$, матричными операторами $\Gamma^{\mu}(27)$ и $\left.R(53),(54)\right)$ и с некоторыми действительными константами $c_{1}$ и $c_{2}$. Данное уравнение, безусловно, можно свести к линейному уравнению со спонтанно нарушенной двойной симметрией, но при этом вновь вводимому полю будет соответствовать представление собственной группы Лоренца, содержащее в своем разложении в прямую сумму неприводимые представления с кратностью не ниже двух. Такие представления были исключены из всех наших прежних исследований в результате учета условия 1 из работы [9]. В пользу сопоставления барионам уравнения (83), на наш взгляд, свидетельствует такая деталь экспериментальной картины нуклонных резонансов [22], как близость по массам низших состояний с противоположными четностями для спинов $5 / 2,7 / 2$ и $9 / 2$. При анализе спектра масс уравнения (83) хотелось бы в первую очередь ответить на вопрос, можно ли получить хорошее согласие с экспериментальными результатами для нуклонов в случае, когда последовательность матричных элементов $\rho(N)$ в формулах $(53),(54)$ знакопеременна (например, когда задается только один параметр $z_{i}$, причем $z_{1}<-2$ ). Если такая возможность реализуема, то можно надеяться на получение падающих с ростом $Q^{2}$ вкладов минимального взаимодействия в электромагнитные формфакторы заряженной частицы, а также на то, что удастся обойтись одним матричным антисимметричным тензорным оператором второго ранга и для заряженной, и для нейтральной частицы.

Благодарности. Я очень признателен С. П. Баранову и В. Е. Троицкому за многочисленные обсуждения проблем, затронутых в настоящей работе.

\section{Список литературы}

[1] I. Estermann, R. Frisch, O. Stern, Nature, 132:3326 (1933), 169-170.

[2] R. W. McAllister, R. Hofstadter, Phys. Rev., 102:3 (1956), 851-856.

[3] D. R. Yennie, M. M. Lévy, D. G. Ravenhall, Rev. Modern Phys., 29:1 (1957), 144-157.

[4] L. H. Hand, D. G. Miller, R. Wilson, Rev. Modern Phys., 35:2 (1963), 335-349.

[5] M. N. Rosenbluth, Phys. Rev., 79:4 (1950), 615-619. 
[6] C. F. Perdrisat, V. Punjabi, M. Vanderhaeghen, Progr. Part. Nucl. Phys., 59:2 (2007), 694-764, arXiv: hep-ph/0612014.

[7] И. М. Гельфанд, А. М. Яглом, ЖЭТФ, 18:8 (1948), 703-733.

[8] И. М. Гельфанд, Р. А. Минлос, З. Я. Шапиро, Представления группы вращений и группь Лорени, их применения, Физматгиз, М., 1958.

[9] Л. М. Сладь, ТМФ, 129:1 (2001), 68-86, arXiv: hep-th/0111140.

[10] Л. М. Сладь, ТМФ, 158:1 (2009), 135-149, arXiv: 0901.3673.

[11] L. M. Slad, Modern Phys. Lett. A, 15:5 (2000), 379-389, arXiv: hep-th/0003107.

[12] M. Gell-Mann, M. Lévy, Nuovo Cimento, 16:4 (1960), 705-726.

[13] Л. М. Сладь, ТМФ, 133:1 (2002), 54-68, arXiv: hep-th/0210120.

[14] Л. М. Сладь, ТМФ, 142:1 (2005), 21-36, arXiv: hep-th/0312150.

[15] V. L. Ginzburg, Acta Phys. Polon., 15:1 (1956), 163-175.

[16] А. А. Комар, Л. М. Сладь, ТМФ, 1:1 (1969), 50-59.

[17] S. Ström, Ark. Fys., 29:39 (1965), 467-483.

[18] T. Janssens, R. Hofstadter, E. B. Hughes, M. R. Yearian, Phys. Rev., 142:4 (1966), 922-931.

[19] L. E. Price, J. R. Dunning, Jr., M. Goitein, K. Hanson, T. Kirk, R. Wilson, Phys. Rev. D, 4:1 (1971), 45-53.

[20] L. M. Slad, Phys. Lett. A, 374:10 (2010), 1209-1213, arXiv: 0904.1671.

[21] V. Bargmann, L. Michel, V. L. Telegdi, Phys. Rev. Lett., 2:10 (1959), 435-436.

[22] C. Amsler, M. Doser, M. Antonelli et al. [Particle Data Group], Phys. Lett. B, 667:1-5 (2008), 1-1339. 